\title{
Profiles of interstellar cloud filaments
}

\section{Observational effects in synthetic sub-millimetre observations}

\author{
M. Juvela, J. Malinen, and T. Lunttila
}

\author{
Department of Physics, PO Box 64, University of Helsinki, 00014 Helsinki, Finland \\ e-mail: mika.juvela@helsinki.fi
}

Received 8 May 2012 / Accepted 11 July 2012

\begin{abstract}
Context. Sub-millimetre observations suggest that the filaments of interstellar clouds have rather uniform widths and can be described with the so-called Plummer profiles. The shapes of the filament profiles are believed to be closely linked to the formation mechanism and physical state of the filaments.

Aims. Before drawing conclusions about the observed column-density profiles, we must evaluate the observational uncertainties. We estimate the bias that could result from radiative transfer effects and from the variations in submm dust emissivity that are expected to take place in dense clouds.

Methods. We use cloud models obtained with magnetohydrodynamic simulations and carry out radiative transfer calculations to produce maps of sub-millimetre surface brightness. Column densities are estimated based on the synthetic observations. For selected filaments, the estimated profiles are compared to those derived from the original column density. The possible effects of spatial variations in the dust properties are examined.

Results. With instrumental noise typical of the Herschel observations, the parameters derived for nearby clouds are correct to within a few percent. The radiative transfer effects have only a minor effect on the results. If the signal-to-noise ratio is degraded by a factor of four, the errors become significant, and for half of the examined filaments the values remain practically unconstrained. The errors also increase in proportion to the cloud distance. Assuming the resolution of Herschel instruments, the model filaments are barely resolved at a distance of $\sim 400 \mathrm{pc}$ and the errors in the parameters of the Plummer function are several tens of percent.

Conclusions. The Plummer parameters, in particular the power-law exponent $p$, are sensitive to noise but can be determined with good accuracy using Herschel data. However, one must be cautious about possible line-of-sight confusion. In our model cloud, a large fraction of the filaments identified from column density maps are discontinuous structures in three dimensions.
\end{abstract}

Key words. ISM: clouds - infrared: ISM - radiative transfer - submillimeter: ISM

\section{Introduction}

For a long time, filaments have been recognised as a common feature of interstellar clouds that is also closely related to the star formation process (e.g., Elmegreen \& Elmegreen 1979; Schneider \& Elmegreen 1979; Bally et al. 1987). Recent Herschel studies and ground-based surveys have revealed the ubiquity of filamentary structures within dense interstellar clouds, and pre-stellar cores and protostars along dense, gravitationally unstable filaments (e.g., Men'shchikov et al. 2010; André et al. 2010; Arzoumanian et al. 2011; Juvela et al. 2012). Therefore, the structure and formation of the filaments are important parts of star formation studies. The filaments are naturally formed as a result of interstellar turbulence (e.g., Padoan $\&$ Nordlund 2011), but significant contribution must exist from immediate triggering by supernova explosions and the radiation and stellar winds from massive stars (e.g., Peretto et al. 2012). The filaments are expected to undergo gravitational fragmentation that will lead to star formation (e.g., Inutsuka \& Miyama 1997; Myers 2009; Heitsch et al. 2011).

Many recent studies have addressed the question of the typical shape of the filament cross-sections. The observed columndensity profiles are fitted with the so-called Plummer function, partly because of the theoretical predictions associated with this functional form. In particular, an infinite isothermal cylinder in hydrostatic equilibrium is expected to follow this profile
(Stodólkiewicz 1963; Ostriker 1964), while, for example, magnetic support, deviations from the isothermal conditions, and initiated collapse would all cause characteristic effects on the parameters of the profile function (see Arzoumanian et al. 2011). If the parameters of the profile function can be determined with high accuracy, this will provide important information on the formation and the current state of the filaments.

The central question is, can we accurately measure the structure and mass of the filaments. Our knowledge is mostly based on molecular lines and dust emission. The kinematic information provided by line observations is crucial but the use of molecules for a structural analysis is complicated by abundance and excitation variations that are difficult to measure and model. The situation is exacerbated in the dense environments where many molecules are severely depleted as they begin to freeze onto dust grains.

Dust emission is considered a reliable probe of dense clouds, especially at sub-millimetre wavelengths where the dependence on the dust temperature is relatively weak. However, the lineof-sight temperature variations can still play a significant role and, in the case of dense cores, can render the column density estimates uncertain by a factor of several (Malinen et al. 2011). Furthermore, dust is expected to undergo evolution in dense and cold environments. This may include accretion of ice mantles, agglomeration of dust particles (leading to the disappearance of small grains), and temperature-dependent changes in the dust 
Table 1. Main parameters of the model clouds.

\begin{tabular}{lccc}
\hline \hline Model & $\begin{array}{c}\left\langle A_{\mathrm{V}}\right\rangle \\
(\mathrm{mag})\end{array}$ & $\begin{array}{c}\left\langle n\left(\mathrm{H}_{2}\right)\right\rangle \\
\left(\mathrm{cm}^{-3}\right)\end{array}$ & Dust model \\
\hline A & 1.0 & 111.0 & standard MWD \\
B & 2.0 & 222.0 & standard MWD \\
C & 4.0 & 450.0 & standard MWD + dust with higher \\
& & & sub-mm opacity \\
\hline
\end{tabular}

Notes. ${ }^{(1)}$ Milky Way dust with $R_{\mathrm{V}}=3.1$.

optical properties (e.g., Ossenkopf \& Henning 1994; Ormel et al. 2011; Meny et al. 2007; Köhler et al. 2011). The analysis of sub-millimetre observations is particularly affected by changes in the dust spectral index $\beta$ and by the possible increase in the dust opacity $\kappa$. The value of $\kappa$ may vary by a factor of several between diffuse and dense regions. This is important for the study of cloud structure, because the derived column densities are directly proportional to $1 / \kappa$. These effects could be expected to be less severe in filaments than in the cloud cores because of the lower density of the filaments and the consequently longer timescale of grain growth. However, significant changes in dust sub-millimetre opacity have been seen also towards filaments. For example, a factor of $\sim 3.4$ increase was reported for a filament in Taurus with $A_{\mathrm{V}}<10^{\mathrm{m}}$ (Stepnik et al. 2003). In addition to the uncertainty in $\kappa$, a wrong assumption about $\beta$ will bias the mass estimates through its effect on the colour temperature estimates (Malinen et al. 2011; Juvela \& Ysard 2012; Ysard et al. 2012).

In this paper, we investigate the possible differences between the real density structure of the filaments and the one deduced from typical sub-millimetre observations of dust emission. The cloud models are based on magnetohydrodynamic simulations with distinct filamentary structures. The emphasis is not on the nature of the filaments themselves but on the uncertainties in deriving their properties from observations. We carry out radiative transfer modelling to produce synthetic surface-brightness maps and estimate the profile functions of selected filaments. The results are compared to the real column-density structure read directly from the model.

The structure of the paper is the following. In Sect. 2, we present the cloud models and the calculations leading to synthetic surface-brightness maps. In Sect. 3, we describe the selected filaments and the procedures used to fit them with Plummer profiles. The main results are shown in Sect. 4, where we list the results for the filament profile fits, both using the true column density and the column density estimated from the synthetic observations. We discuss the results in Sect. 5 before presenting our final conclusions in Sect. 6.

\section{Simulated clouds and observations}

The modelling is based on a magnetohydrodynamic (MHD) simulation carried out on a regular grid of $1000^{3}$ cells. The root mean square (rms) sonic Mach number is $\sim 9$ and the rms Alfvénic Mach number 2.8. The data are the same as those used in Malinen et al. (2011) and we refer to that paper for details. The MHD calculations assume an isothermal equation of state. The simulation was run first without gravity and the stirring continued for several shock crossing-times to develop turbulence wellseparated from the initial conditions. The simulation was continued with gravity switched on and the data used here corresponds to the situation during early collapse (model I in Malinen et al. 2011). We use the unigrid run (Padoan $\&$ Nordlund 2011) rather than the adaptive mesh refinement (AMR) MHD data because we need high spatial resolution not only for the cores but also for the lower density filaments.

The cloud size was scaled so that the mean visual extinction $A_{\mathrm{V}}$ through the cloud is $1.0^{\mathrm{m}}$ or $2^{\mathrm{m}}$. In the following, these are called models A and B. Assuming a linear cloud size of $6 \mathrm{pc}$, these correspond to mean hydrogen densities of $111 \mathrm{~cm}^{-3}$ and $222 \mathrm{~cm}^{-3}$, respectively.

For the radiative transfer calculations, we built an adaptive grid from the $1000^{3}$ full resolution model. The adaptive grid is constructed by combining neighbouring cells that are optically thin, and have similar densities. The maximum allowed $V$-band optical thickness of a cell is $\tau_{\max }=0.04$ in model A. The maximum density contrast was set to $\rho_{\max } / \rho_{\min }=30$. The resulting adaptive model has approximately $160 \times 10^{6}$ cells and consists of 839 grids. The same grid was used for model B. The gridding ensures that moderately dense regions, the filaments in particular, are also sampled at the best resolution.

We use the radiative transfer program described in Lunttila \& Juvela (2012; see also Juvela \& Padoan 2003; Juvela 2005). The cloud is illuminated externally by the isotropic interstellar radiation-field (Mathis et al. 1983). The dust is assumed to have the properties of the standard Milky Way diffuse interstellar dust $\left(R_{\mathrm{V}}=3.1\right)$ described in Draine (2003). Because of the low dust temperatures and the modest optical thickness of the model clouds, the absorption of the thermal dust emission does not contribute significantly to the dust heating. Therefore, the dust temperature was calculated in a single step without iteration.

In the third model $\mathrm{C}$, the average visual extinction is set to $A_{\mathrm{V}} \sim 4^{\mathrm{m}}$. Assuming a linear cloud size of $6 \mathrm{pc}$, the mean density is $n(\mathrm{H})=450 \mathrm{~cm}^{-3}$. In model C, we use two dust components. The second one corresponds to a modification where the dust sub-millimetre opacity is increased. This is implemented by multiplying the original opacities with a factor $(\lambda / 20 \mu \mathrm{m})^{0.5}$ at wavelengths longer than $20 \mu \mathrm{m}$. The resulting dust-opacity spectral index is thus $\beta \sim 1.5$ in the sub-millimetre and, as a result, the sub-millimetre opacity $\kappa_{v}$ is higher than for the unmodified dust component. For example, at $250 \mu \mathrm{m}$ the $\kappa_{v}$ has increased by a factor of 3.5. The relative abundance of the two dust components is varied according to the local density so that the abundances are equal at a density of $n(\mathrm{H}) \sim 5 \times 10^{4} \mathrm{~cm}^{-3}$ (see Fig. 5 in Malinen et al. 2011). At low densities, the relative abundance of the modified dust component goes to zero. By the time the density reaches $10^{6} \mathrm{~cm}^{-3}$, practically all the dust is in the modified form. The calculations for model $\mathrm{C}$ were carried out at a resolution of $500^{3}$ cells where the grid was obtained by direct downsampling the original $1000^{3}$ data.

The radiative transfer calculations (Juvela \& Padoan 2003; Lunttila \& Juvela 2012) solve the three-dimensional (3D) temperature structure of the model clouds and result in synthetic surface-brightness maps of $1000 \times 1000$ (models A and B) or $500 \times 500$ pixels (model C). Surface brightness maps were calculated at $160,250,350$, and $500 \mu \mathrm{m}$ which correspond to the wavelengths of Herschel observations. The full-resolution images were convolved with Gaussian beams of full width at half maximum (FWHM) corresponding to 3, 6, or 12 pixels (in model $\mathrm{C}$, the beam widths in pixels are half of these values). Before the convolution, we add to the maps white noise that corresponds to $3.7,1.20,0.85,0.35 \mathrm{MJy} / \mathrm{sr}$ per original Herschel beam size of $12,18,25$, and $37^{\prime \prime}$, respectively. The values were 

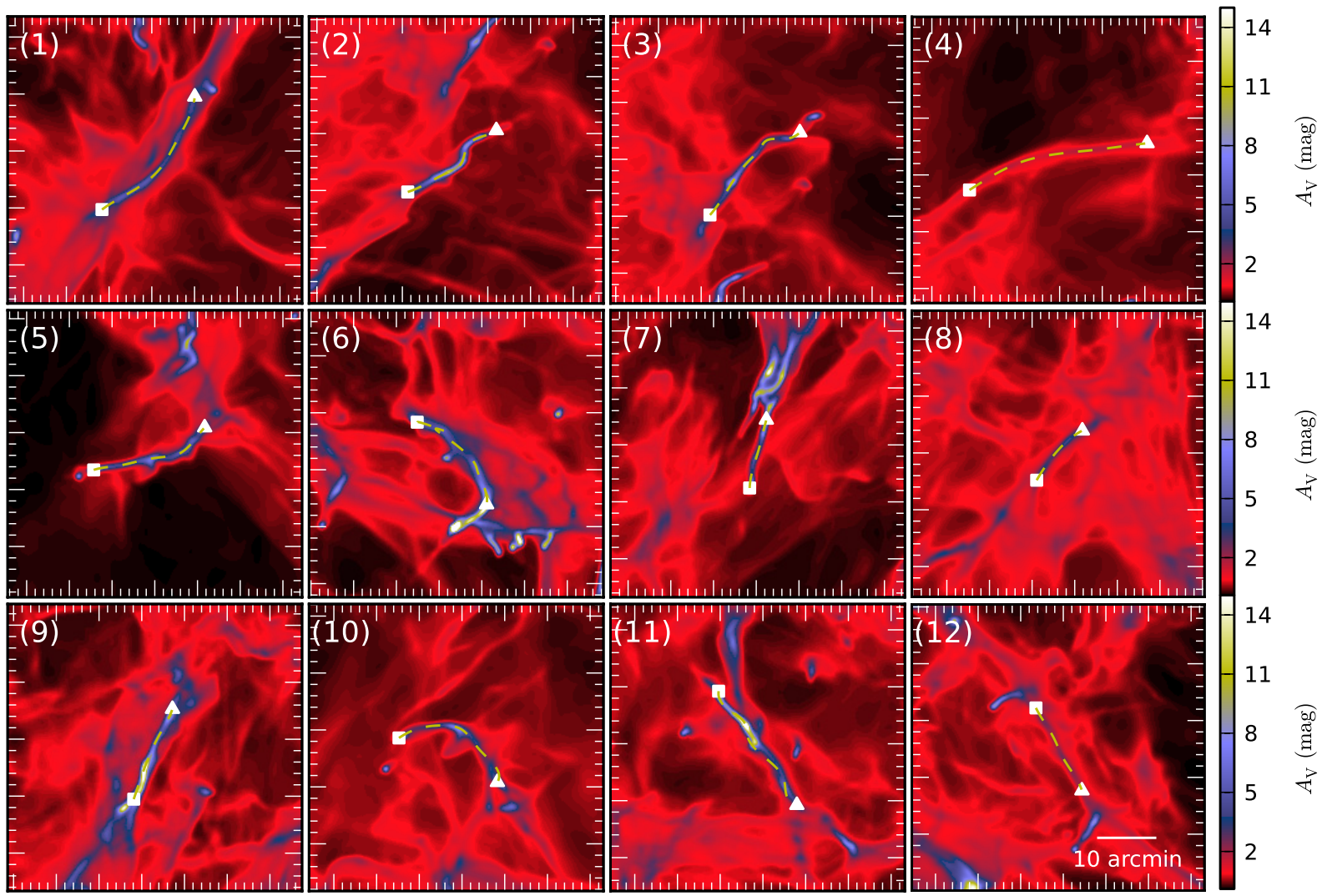

Fig. 1. $A_{\mathrm{V}}$ maps of the selected twelve filaments in model A. The dashed lines indicate the filament segments included in the analysis. The $A_{\mathrm{V}}$ values are obtained directly from the model cloud where the $1000 \times 1000$ pixel image has been convolved with a beam of FWHM equal to three pixels.

obtained using the Herschel observations planning tool HSPOT ${ }^{1}$ and they correspond to the instrumental noise levels of typical Herschel mapping observations (as in Malinen et al. 2011). Most actual Herschel observations are confusion-limited, particularly at wavelengths $\lambda \geq 250 \mu \mathrm{m}$. In our simulations, the confusion results only from structures inside the modelled volume. In more realistic simulations, especially for low surface-brightness values, one might also have to consider the confusion by extragalactic point sources. The final common resolution of all the maps (i.e., the size of the beam used in the convolution) is $40^{\prime \prime}$. When the data are convolved with a beam equal to 3 pixels, the pixel size is thus $13.3^{\prime \prime}$ and the map size is 3.7 degrees. With the assumed cloud size of $6 \mathrm{pc}$, the FWHM beam sizes of 3,6, and 12 pixels translate to the cloud distances of 93,186 , and $371 \mathrm{pc}$ (in the following these are called the near, medium, and far distances). For the near distance, the filaments are clearly larger than the beam. For the medium distance, the filaments are still slightly larger than the beam, but in the case of the far distance, the observed filament width is dominated by the convolution.

\section{Methods of analysis}

The synthetic surface-brightness observations were converted to maps of column density. The dust colour temperature was calculated pixel by pixel by fitting the observed 160, 250, 350, and $500 \mu \mathrm{m}$ intensities with a modified black-body curve. The data points were weighted according to the noise levels of the simulation. In the fits, the opacity spectral index was fixed to a constant value of $\beta=1.8$ or $\beta=2.0$. These values differ from the

\footnotetext{
${ }^{1}$ http://herschel.esac.esa.int/Tools.shtml
}

actual value in the dust model (Milky Way dust with $R_{\mathrm{V}}=3.1$ ), where the spectral index between the wavelengths of $160 \mu \mathrm{m}$ and $500 \mu \mathrm{m}$ is 2.09 .

With the dust colour temperature known, the column density is calculated as

$$
N^{\mathrm{Obs}}=\frac{S_{v}}{B_{v}\left(T_{\mathrm{C}}\right) \kappa_{v}}
$$

where $S_{v}$ is the intensity of the fitted modified black-body curve, $B_{v}$ is the Planck function, and $\kappa_{v}$ is the dust opacity. The $\kappa_{v}$ value was taken directly from the dust model. Therefore, the derived column density $N^{\text {Obs }}$ differs from the true column density $N^{\text {True }}$ only because of noise and systematic errors in the colour temperature $T_{\mathrm{C}}$, the latter being caused by line-of-sight temperature variations.

We selected four filaments from each of the three columndensity images calculated towards three orthogonal directions (see Fig. 1). The filament segments were selected by eye and the path of each filament was set to follow the peak of the filament as seen in the $N^{\text {True }}$ maps obtained from the cloud model.

The procedure used in analysing the filaments is basically the same as in Juvela et al. (2012). The column density profiles perpendicular to the filament path were extracted at $20^{\prime \prime}$ intervals. The individual profiles were aligned so that their peaks match before calculating the median profile of the whole filament. Thus, the location of the filament was allowed to differ from the originally selected path. A fixed range of $[-0.3 \mathrm{pc}$, $+0.3 \mathrm{pc}]$ around the peak of the column density profile was included in the subsequent analysis. The filament FWHM was measured by fitting the profile with a Gaussian function plus a 
constant background. The filament FWHM values were determined for each cross-section separately and for the final combined median profile. The median profile of each filament was also fitted with a model consisting of a linear background plus a Plummer-like column-density profile

$N(r)=A_{p} \frac{\rho_{\mathrm{c}} R_{\mathrm{flat}}}{\left[1+\left(r / R_{\mathrm{flat}}\right)^{2}\right]^{(p-1) / 2}}$

(see Arzoumanian et al. 2011; Nutter et al. 2008; Juvela et al. 2012). The factor $A_{p}$ is calculated using the formula $A_{p}=$ $\int_{-\infty}^{\infty}\left[\left(1+u^{2}\right)^{p / 2} \cos i\right]^{-1} \mathrm{~d} u$, where we assume an inclination angle of $i=0$ (Arzoumanian et al. 2011). In addition to the two parameters describing the background, the free parameters of the Plummer function are the central density of the filament $\rho_{\mathrm{c}}$, the size of the inner flat part $R_{\text {flat }}$, and the power-law exponent $p$. The finite resolution of the observations was taken into account by fitting the observed profile not directly with the theoretical Plummer profile but with a Plummer profile convolved with the beam used in the simulated observations. The convolution weights take into account that the actual convolution takes place over a two-dimensional map. This allows us to derive deconvolved parameters for even unresolved filaments.

In addition to the fitted parameters, we also look at some derived quantities. In particular, the mass per unit length of filament, $M_{\text {line }}$, can be calculated by integrating over the fitted Plummer profile.

\section{Results}

The twelve filaments were analysed using the true column density $N^{\text {True }}$ and the column densities $N^{\text {Obs }}$ estimated from synthetic observations. The analysis was repeated with an actual resolution equal to 3,6 , and 12 pixels of the original $1000 \times 1000$ pixel maps, corresponding to the near, medium, and far cloud distances. Owing to the lower resolution of the model $\mathrm{C}$, these correspond to convolution with a beam with a FWHM equal to $1.5,3$, and 6 pixels, respectively.

Figure 2 shows examples of the column-density profiles, comparing the true column density profiles to those estimated from the synthetic observations. These already indicate that in models $\mathrm{A}$ and $\mathrm{B}$ the column densities are underestimated by $\sim 10 \%$ at the centre of the filament, because the estimated colour temperature $T_{\mathrm{C}}$ overestimates the mass-averaged dust temperature. That the analysis is carried out with a slightly too low value of the spectral index, $\beta=2.0$, instead of 2.09 , contributes in the same direction but is a smaller factor. In model $\mathrm{C}$, the submillimetre dust opacity was increased by a factor of a few in the densest parts of the cloud. This is directly reflected in the column density estimates that are more than twice the correct value at the centre of the filaments. The figure also shows that, apart from the filament itself, the profiles are affected by other emission components, i.e., by background confusion.

Each filament was fitted with a Plummer function. Figure 3 shows the results for model $\mathrm{A}$ in the case where the observed column densities were estimated with $\beta=2.0$ and the cloud distance was set to $d=93 \mathrm{pc}$, which corresponds to convolution with a beam of FWHM equal to 3 pixels. The figure demonstrates that the profiles can be well-fitted using the Plummer function. In Fig. 3, the parameter values show a large scatter. Malinen et al. (2012) noted that the fitting of Plummer profiles is sometimes sensitive to the initial parameter values of the least squares minimisation. Visual inspection shows that all the fits in

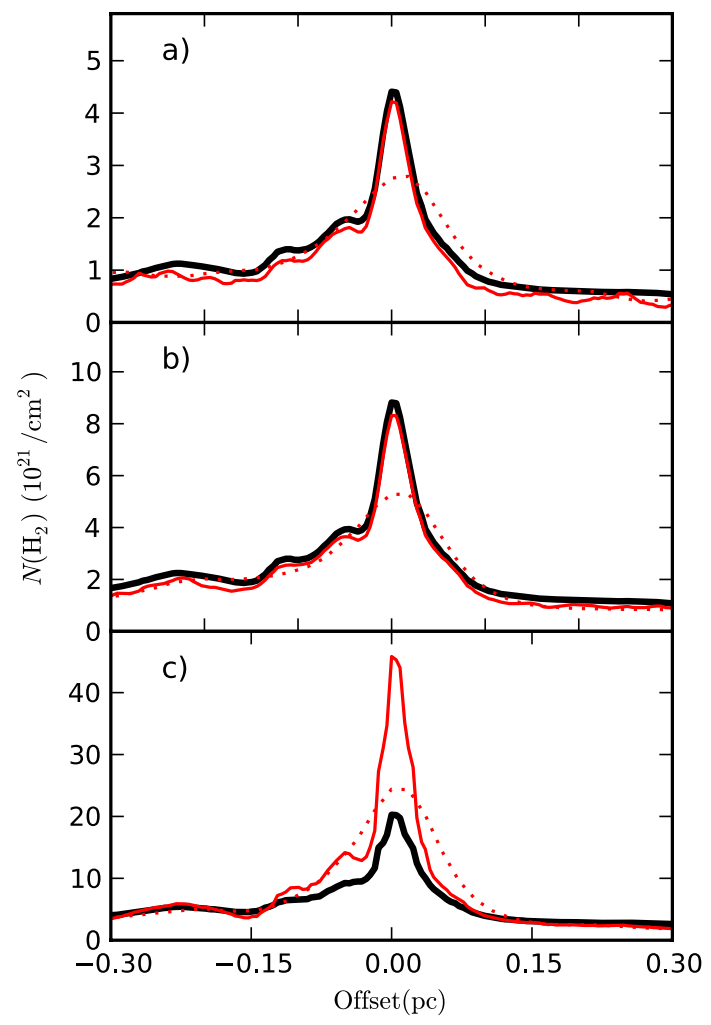

Fig. 2. The profile of the filament number 1 as seen in the models A-C (frames a)-c), respectively). The solid thick line shows the actual column-density profile in the model cloud. The other curves show the profiles derived from the synthetic observations when the cloud is at a near distance of $93 \mathrm{pc}$ (thin solid line) or $371 \mathrm{pc}$ (dotted line).

Fig. 3 are good. However, in addition to intrinsic variations between the filaments, some of the scatter is caused by the instrumental noise and especially by the confusion caused by other emission along the line-of-sight.

Figure 4 shows a comparison of the estimated values of $\rho_{\mathrm{c}}$, $p$, and $M_{\text {line }}$ for all three beam sizes (3, 6, and 12 pixels) for model A. On the $x$ axis, we plot the parameters obtained using the true column densities, and on the $y$ axis the values derived from the analysis of synthetic observations with and without observational noise and with $\beta$ equal to either 1.8 or 2.0 . The effect of the $\Delta \beta=0.2$ is relatively small and is seen mainly as a systematic effect in the density $\rho_{\mathrm{c}}$ and mass $M_{\text {Line }}$ values where it amounts to an underestimation of 20-30\%. Without noise, the parameter values obtained using the observed and true column densities are similar to within a few percent. The effect of noise is often small but can also lead to tens of percent differences in the parameter values. The loss of resolution (i.e., convolution with a larger beam) has a clear impact when noise is included and leads to a larger scatter especially in the estimates of the central density, which are underestimated in most cases, by even up to $\sim 2$ orders of magnitude. In the fitting routine, the maximum allowed value for the parameter $p$ is 10 . The largest bias in $p$ values occurs when the value derived from either the true or observed column density (but not both) is $\sim 10$. Compared to the other parameters (especially the central density $\rho_{\mathrm{c}}$ ), the mass $M_{\text {Line }}$ is less affected by the noise and the resolution.

The corresponding results for the higher column-density model B are presented in Fig. 5. We also note that the parameter values obtained using the observed and true column densities 
M. Juvela et al.: Profiles of interstellar cloud filaments

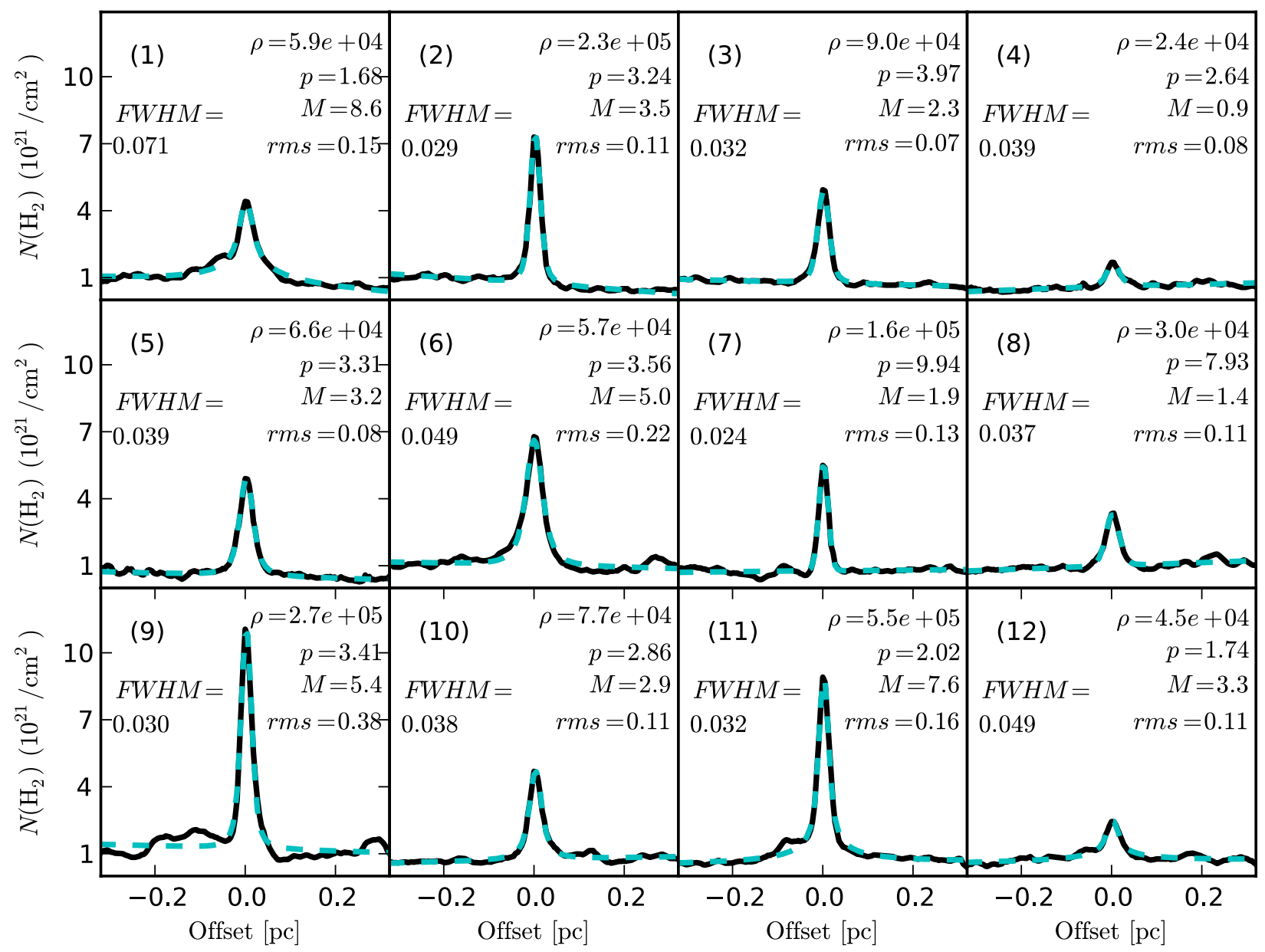

Fig. 3. Fits of the Plummer function to profiles extracted from the observed column-density maps $(\beta=2.0)$ of model A cloud at the near distance. The solid black curves show the estimated median profiles of the filaments and the dashed cyan lines the fitted functions. The parameters $\rho_{\mathrm{c}}$ (central number density of $\mathrm{H}_{2}$ in units of $\mathrm{cm}^{-3}$ ), $p$, and $M_{\text {line }}$ (in units of $M_{\odot} / \mathrm{pc}$ ) are shown in the frames, as well as the rms value of the fit (in units of $10^{21} \mathrm{~cm}^{-2}$ ) and the FWHM values of the filaments obtained from fits with Gaussian functions.

without instrumental noise are in most cases similar to within a few percent.

The scatter in the central density $\rho_{\mathrm{c}}$ values (from $N^{\mathrm{Obs}}$ including noise vs. those from $N^{\text {True }}$ ) appears to be smaller than for model A. Using the observations with beam sizes equal to 3 and 6 pixels (corresponding to the near and medium cloud distances) the density and mass are mostly either correct or somewhat underestimated. However, when low resolution with a beam size of 12 pixels is used, the scatter increases and the parameters can be also overestimated. For the $M_{\text {Line }}$ values, the error is up to a factor of $\sim 2$. We note that this is the error between the masses derived from either the true or the estimated column density. We return later to the comparison with the actual mass of the filaments that can be obtained directly from the model cloud.

In model $\mathrm{C}$, the abundance of modified dust, i.e., the component with a higher sub-millimetre opacity, increases with density. Figure 6 shows the relative abundance of the modified dust as projected values in three orthogonal directions. The locations of the selected filaments are marked in the figure. The figure shows that, while modified dust is the dominant component towards the centre of many filaments, its abundance remains below $20 \%$ in most of the area included in the Plummer fits, i.e., at distances of a few arcmin from the filament centre. Thus, the spatial variation in the dust properties should be able to bias both the $N_{\text {Obs }}$ values and the observed shape of the profiles, and hence affect the parameters of the Plummer fits.

Figure 7 presents some examples of the column density profiles of the model $\mathrm{C}$ filaments, comparing $N^{\mathrm{Obs}}$ and $N^{\text {True }}$. Because the column densities were estimated assuming a constant dust opacity, $\kappa_{v}$, the column densities were overestimated whenever the dust opacity had been increased. In the figure, the $N^{\text {True }}$ values have been scaled by a factor of 1.5 . This provides rough agreement with $N^{\mathrm{Obs}}$ in the outer parts of the filaments, while at the centre the synthetic observations lead to still higher column densities by a factor of $\sim 2$. For example, the effect on the observed filament FWHM depends on whether the emission at the half value point is produced mainly by the normal dust or the modified dust. In the latter case, the observed filament FWHM would not be affected, even though the column density can be strongly overestimated.

The values of the Plummer parameters obtained with model $\mathrm{C}$ are shown in Fig. 8. The optical depth of the cloud is about twice as high as in the case of model B. Therefore, in addition to the spatial variation in the dust properties, the radiative transfer effects also should have a stronger effect on the results. The observations are seen to overestimate the filament mass by up to a factor of several. Thus, the increase in the average $\kappa_{v}$ dominates over the opposite effect resulting from the 

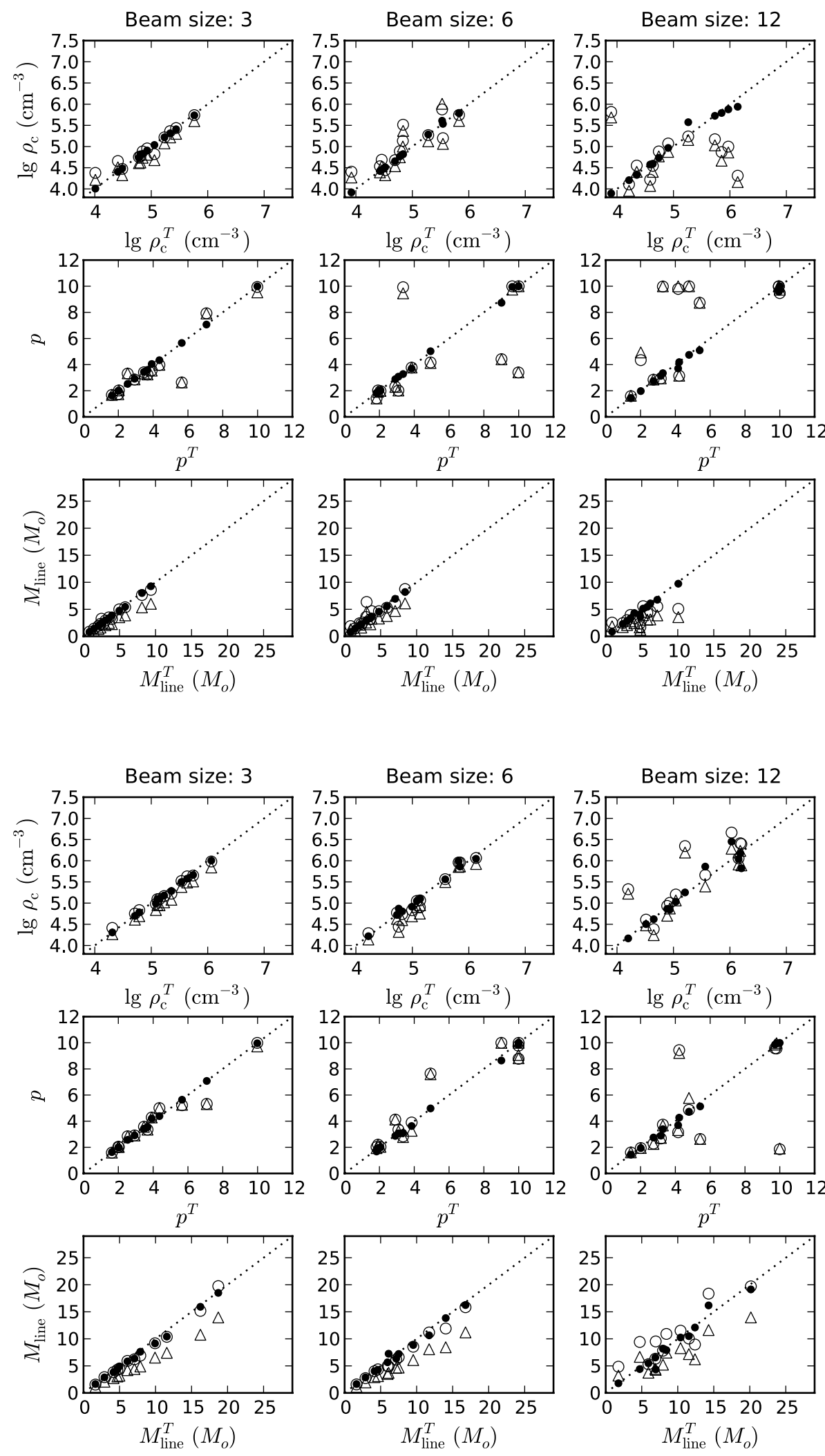

Fig. 4. Comparison of the Plummer parameters derived from true column density and the values estimated from the synthetic maps of model A. The rows correspond to parameters $\rho_{\mathrm{c}}, p$, and $M_{\text {line }}$. The columns correspond to beam sizes equal to 3,6 , and 12 pixels, i.e., to the near, medium and far cloud distances. On the $x$-axis, we always plot the parameter values from $N^{\text {True }}$ and on the $y$-axis the values from $N^{\mathrm{Obs}}$ derived with $\beta=2.0$ and without observational noise (solid circles), with $\beta=2.0$ and noise (open circles), and with $\beta=1.8$ and noise (triangles).
Fig. 5. As Fig. 4, but for the model B that has twice as high a column density. dispersion of dust temperatures along the line of sight. The volume density estimates can be $\sim 0.5-1$ orders of magnitude too high and are more biased when the resolution of the observations is low. The scatter is larger than in the previous models, especially when the data are convolved with the largest 12 pixel beam. The smaller spectral index $\beta=1.8$ still leads to lower
$M_{\text {Line }}$ values but the scatter in the values suggests that the fits are less well-constrained. The $p$ values can be divided into two groups: those close to $p \sim 10$ and those with values $p \sim 2-4$. At low $p$, the values obtained from synthetic observations do not show any systematic bias. In contrast to models $\mathrm{A}$ and $\mathrm{B}$, in model $\mathrm{C}$ there are also cases without instrumental noise where 

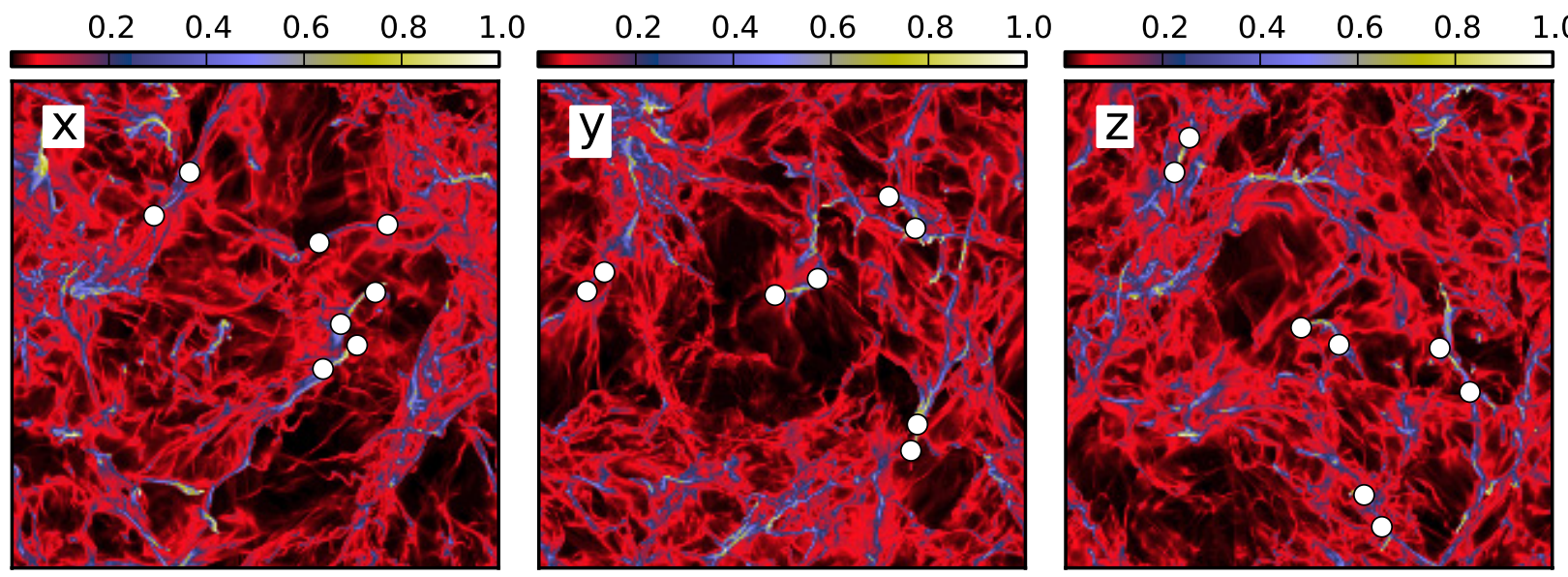

Fig. 6. The fractional abundance of modified dust in the model C. The three frames correspond to three orthogonal directions of observations. The white circles indicate the ends of the filament segments included in the analysis.

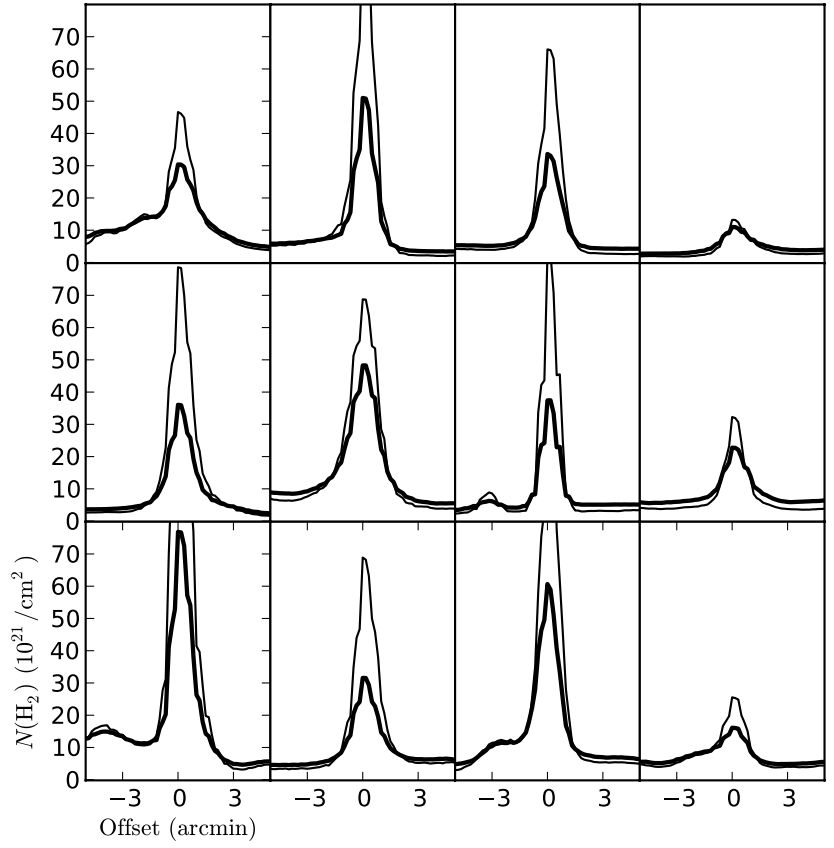

Fig. 7. Profiles of the filaments in model $\mathrm{C}$ (at the near cloud distance). The thick lines correspond to the true column density $N^{\text {True }}$ of the model cloud multiplied by a factor of 1.5. The thin lines denote the column density derived from synthetic observations.

the $p$ value derived from either the observed column density or the true column density (but not both) is close to 10 .

The FWHM values of the filaments were calculated with Gaussian fits and Fig. 9 compares the values in the three models. The values derived from $N^{\text {True }}$ should be identical in all three models. However, because of the coarser initial resolution of model $\mathrm{C}$, the values of that model differ slightly from those of the others. In models $\mathrm{A}$ and $\mathrm{B}$, the values obtained from synthetic observations are always within a couple of percent of the $N^{\text {True }}$ results. In model B, the filament optical depths are above $A_{\mathrm{V}}=10^{\mathrm{m}}$ and one could expect the central column density to be underestimated relative to the lower density outer regions. However, the effect on the beam convolution is still almost negligible. More dispersion is seen in model C. Because the enhanced emissivity increases the signal at the centre of the filaments, the estimated FWHM values of the filaments is expected to be smaller. This is also the observed trend, the analysis of $N^{\text {Obs }}$ leading to $\sim 10-20 \%$ smaller values than $N^{\text {True }}$. The effect is not quite constant, probably because of the additional effect of instrumental noise and the confusion by dense background structures. The largest differences are $\sim 40 \%$ (filaments number 1 and 12).

We compare the $p$ values of the twelve filaments in the three models in Fig. 10, using the medium cloud distance of $186 \mathrm{pc}$ (beam size equal to 6 pixels) and $\beta=2.0$. When the parameter values are derived from the true column density, $N^{\text {True }}$ (frame $c$ ), all the models give similar results, regardless of the value. In four out of twelve cases, the $p$ value is above 8 , indicating that the parameters are not well-constrained, i.e., the minimum $\chi^{2}$ value is reached with parameter values that are far from those obtained in the absence of noise. Most of the other twelve filaments have $p$ values in the range $2-4$. When the parameter values are derived from the observed $N^{\mathrm{Obs}}$ without instrumental noise (frame $a$ ), the results are mostly similar (to within $\Delta p \sim 0.5$ ) to the parameter values derived from the true $N^{\text {True }}$. Only in two cases is the $p$ value in model $\mathrm{C}$ biased by a factor of $\sim 2$, notably to opposite directions. However, when using the observed $N^{\text {Obs }}$ with noise (frame $b$ ), the situation gets more complicated. Now only half of the filaments have similar $p$ values (to within $\Delta p \sim 0.5$ ) as in the case for the true $N^{\text {True }}$. This applies to all three models. The bias can now also be seen in all models, particularly model A. These results indicate that the effect of instrumental noise on the derived $p$ parameter is more prominent than the radiative transfer effects.

The results of Figs. 4, 5, and 8, are summarised in Fig. 11. The figure shows for the three model clouds and the three cloud distances the distributions of the relative parameter errors using boxplots ${ }^{2}$. The errors are calculated as the difference between the values derived from $N^{\text {Obs }}$ (with $\beta=2.0$ ) and $N^{\text {True }}$, normalised with the latter parameter estimate. The figure includes the error distributions for the logarithm of the central density $\rho_{\mathrm{c}}$, parameter $p$, and the mass $M_{\text {line }}$.

${ }^{2}$ See http://en.wikipedia.org/wiki/Box_plot 

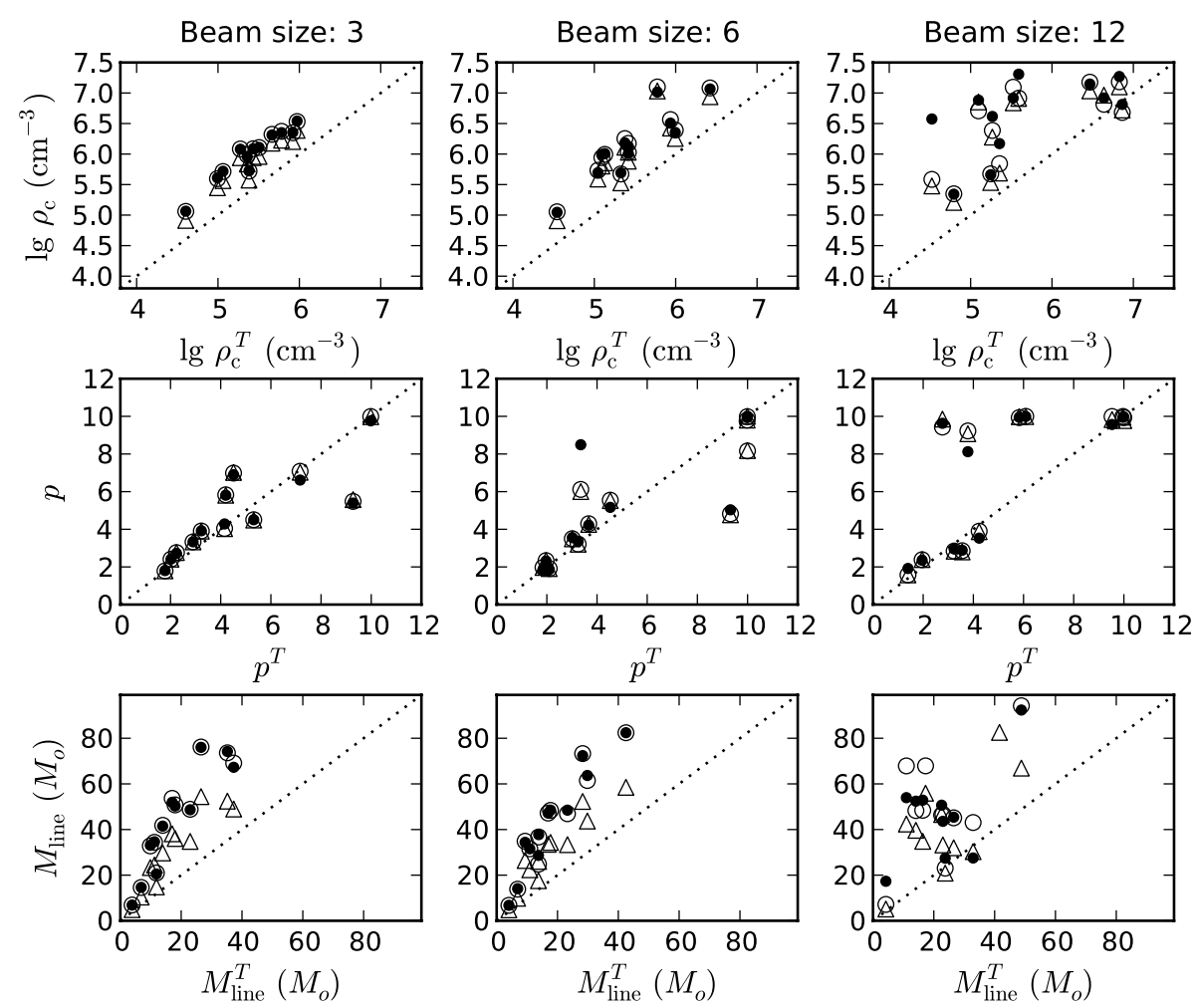

Fig. 8. As Fig. 4, but for model C containing two dust components with a spatially varying abundance ratio. The beam size $(3,6$, or 12 pixels corresponding to the near, medium, or far cloud distance, respectively) is indicated on top of each column of frames.

\section{Discussion}

The estimated central density of the filament $\rho_{\mathrm{c}}$, the power-law exponent $p$, and the mass per length unit $M_{\text {Line }}$ were examined in Sect. 4. We have shown that the filaments of our MHD model can be well-fitted with the Plummer function. However, the fitting is sometimes sensitive to the initial values of the fitting parameters (not all initial values leading to a good fit) and the parameters extracted from apparently good fits can have a large scatter, especially in the case of filaments that are not clearly resolved. This indicates that the parameters depend on the amount of instrumental noise added and thus very likely on the confusion with background structures. All the fits used in this paper visually look good, i.e., the least squares procedure appears to have converged to an apparently valid solution. However, different initial values might lead to slightly different combinations of parameters values because, for example, $R_{\text {flat }}$ and $p$, are intrinsically correlated. Malinen et al. (2012) also noted that small changes in the input data can sometimes have a large effect on the estimated Plummer parameters.

In models $\mathrm{A}$ and $\mathrm{B}$, when observational (i.e., instrumental) noise was not included, the parameters derived from $N^{\text {True }}$ and $N^{\text {Obs }}$ were similar to within a few percent. Furthermore, in the case of the near distance cloud (beam size equal to 3 pixels), the results did not depend on the level of instrumental noise. When the resolution of the observations was poor, in particular for the far cloud distance of $371 \mathrm{pc}$ corresponding to convolution with a beam of FWHM equal to 12 pixels, noise did cause significant scatter. In the central density $\rho_{\mathrm{c}}$ the errors could occasionally increase by up to two orders of magnitude. In this case, the beam is larger than the width of the filament and the observations provided weak constraints on the central profile. Occasionally, the result is a very narrow and high density peak that, when convolved with the large beam, is still consistent with the observations.

Compared to model A, model B was much less affected by the noise because of its higher column density and thus higher signal-to-noise ratios. In model $\mathrm{C}$, with modified dust, central density was overestimated by up to $\sim 0.5-1$ orders of magnitude, or even more with the lowest resolution. Similarly, the mass estimates could be incorrect by a factor of several. The scatter was much larger than in the models A and B, especially for the medium and far cloud distances. For the clouds at the far distance (lowest resolution), the total width of the profile is dominated by the convolution and the values of the derived Plummer parameters are more uncertain. This is clearly demonstrated by Fig. 11. The typical errors increase by a factor of several between the near and far distances. On the other hand, in the case of model B the errors are only half of those found in model A. For model $\mathrm{C}$, the errors are larger. The mass estimates are directly affected by the wrong assumption made about the dust opacity but the other parameters do not appear to be very significantly biased.

The central question is, are the fitted parameter values, in particular $p$, so reliable that theoretical conclusions can be made based on them. In all our models, the $p$ parameter values have a bipolar behaviour. The lower values $(\sim 2-4)$ are mostly within a few percent of those derived from the true column density. The largest errors occur when the $p$ value derived from either the observed or true column density is near the maximum allowed value of 10 . In models $A$ and $B$, this occurs only when instrumental noise is included, but in model $\mathrm{C}$ also in the absence of noise. Noise and low resolution clearly increase the probability of getting a high value for $p$, which implies that the parameters are not well-constrained. Malinen et al. (2012) demonstrated that the confidence regions of the Plummer parameters can be wide and the parameters (e.g., $p$ and $R_{\text {flat }}$ ) strongly correlated. We also compared the $p$ parameter values derived from our three models for individual filaments (Fig. 10). The effect of noise appears to be much stronger than the radiative transfer effects.

To separate the effects of instrumental noise from those of spatial resolution, we carried out tests where only the noise levels were varied. Figure 12 shows the results for model $\mathrm{A}$ at the 


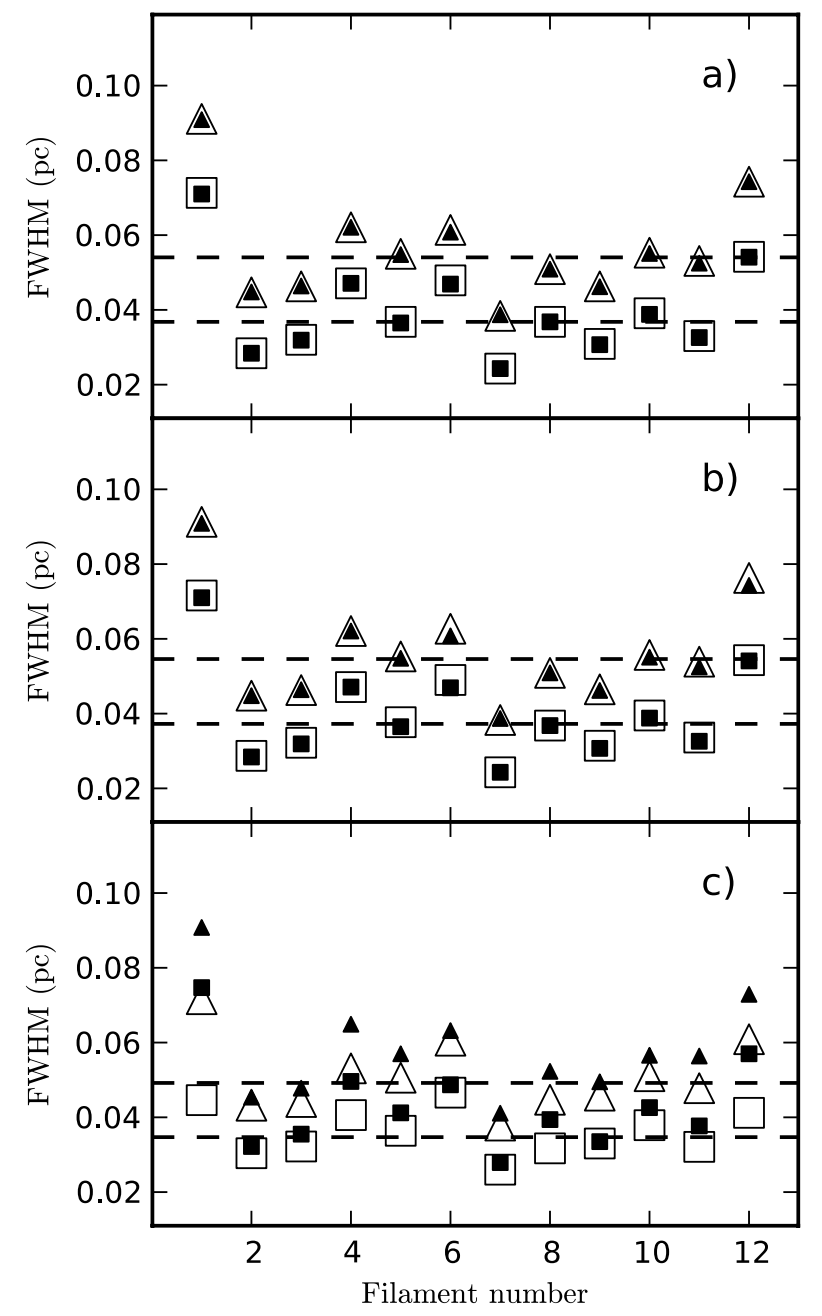

Fig. 9. The FWHM values of the twelve filaments. The frames a)-c) correspond to the models $\mathrm{A}-\mathrm{C}$, respectively. The squares and the triangles correspond to the near cloud distance of $93 \mathrm{pc}$ and the medium cloud distance of $186 \mathrm{pc}$, respectively. The solid symbols are values derived from $N^{\text {True }}$ and the open symbols values from $N^{\text {Obs }}$. The dashed lines indicate the median values for the FWHM values derived from $N^{\text {Obs }}$. Upper line corresponds to the distance of $186 \mathrm{pc}$ and lower line to the distance of $93 \mathrm{pc}$.

near distance. The noise is scaled between 0.5 and 4.0 times the values used above. The figure shows the median parameter values and their inter-quartile ranges for each filament and each noise level, as determined from 50 noise realisations. With the default noise, all the parameters shown are still well-determined. When the noise is increased, the scatter in the parameters derived from the noise realisations increases rapidly. The noise increase also causes a bias in the case of some filaments. However, this varies from filament to filament and, for example, the median value of $M_{\text {line }}$ can either increase or decrease. The figure also implies that as the cloud distance is increased, part of the increase in the scatter in the parameter values is caused by noise. At the far distance, the apparent size of the filament is four time smaller than at the near distance and the total signal-to-noise ratio (integrated over the source) is correspondingly smaller. At the relative noise value of 4.0 the value of $p$ is well-constrained (to within 50\%) for fewer than half of the selected filaments. In the fits, $p$ was always restricted to values below 10.0.

We have concentrated on the differences between the true and the observed column densities and their effect on the

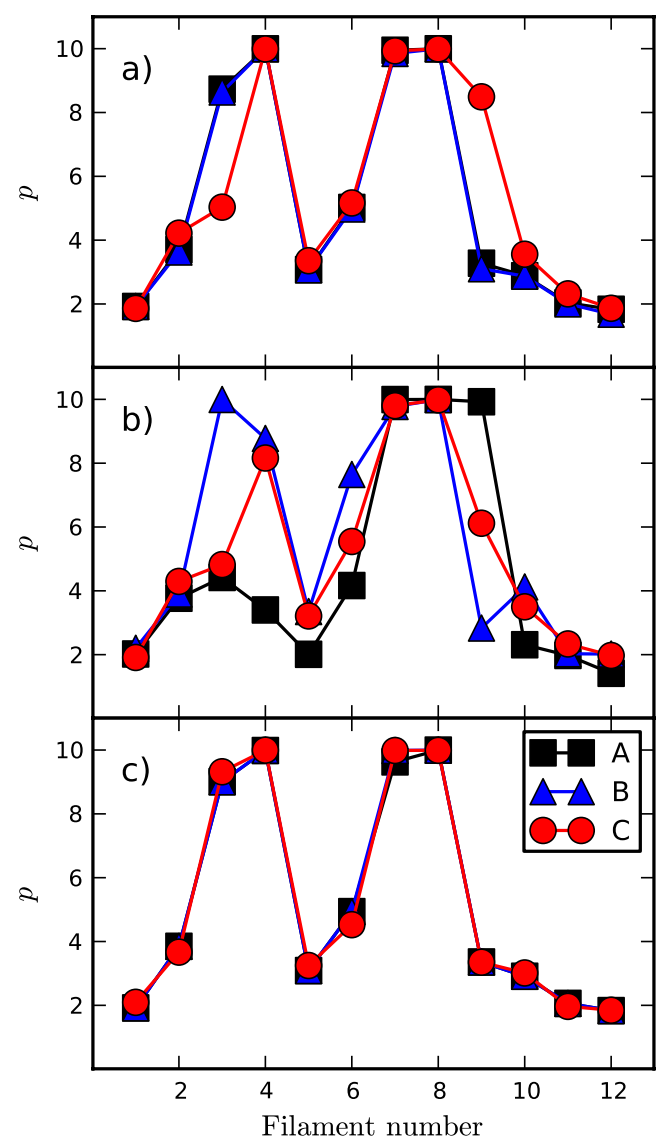

Fig. 10. The $p$ values of the twelve filaments using medium cloud distance of $186 \mathrm{pc}$ (beam size of 6 pixels) and $\beta=2.0$. Squares indicate model A, triangles model B, and circles model C. a) Values are derived from the observed $N^{\mathrm{Obs}}$ without noise. b) Values are derived from the observed $N^{\mathrm{Obs}}$ with noise. c) Values are derived from the true $N^{\text {True }}$.

estimated filament parameters. However, we can note some additional factors affecting the interpretation of these parameters. As in our models, the line-of-sight confusion can be a problem in real observations. For example, some of the occasionally large values of the parameter $p$ are suspected to be caused by background structures. Those can be confused with the filament itself or they may affect the fits by modifying the background estimates (see Fig. 3).

The line-of-sight confusion can be even more severe and some of the identified filaments can be the result of a chance alignment of unrelated (or related but still physically separate) density structures. Of the four filaments selected from the $x$ direction maps, none is seen as a clear isolated filament when examined from the other two orthogonal directions. Figure 13 illustrates this in the case of filament 1 . The filament was identified from the image towards the direction $x$. We determined the locations of the volume density maxima for a few positions along the filament along the line of sight. The images calculated for the other two orthogonal viewing-directions show that the filament is actually a superposition of at least two independent density structures. This may also be a common scenario in the case of real clouds, as suggested, for example, by the identification of several kinematically separate components in some of the Taurus filaments (Tafalla 2010, priv. comm.).

Figure 14 shows the central densities of filaments derived from the observations. For comparison, the figure includes the actual maximum line-of-sight density that is obtained from the 


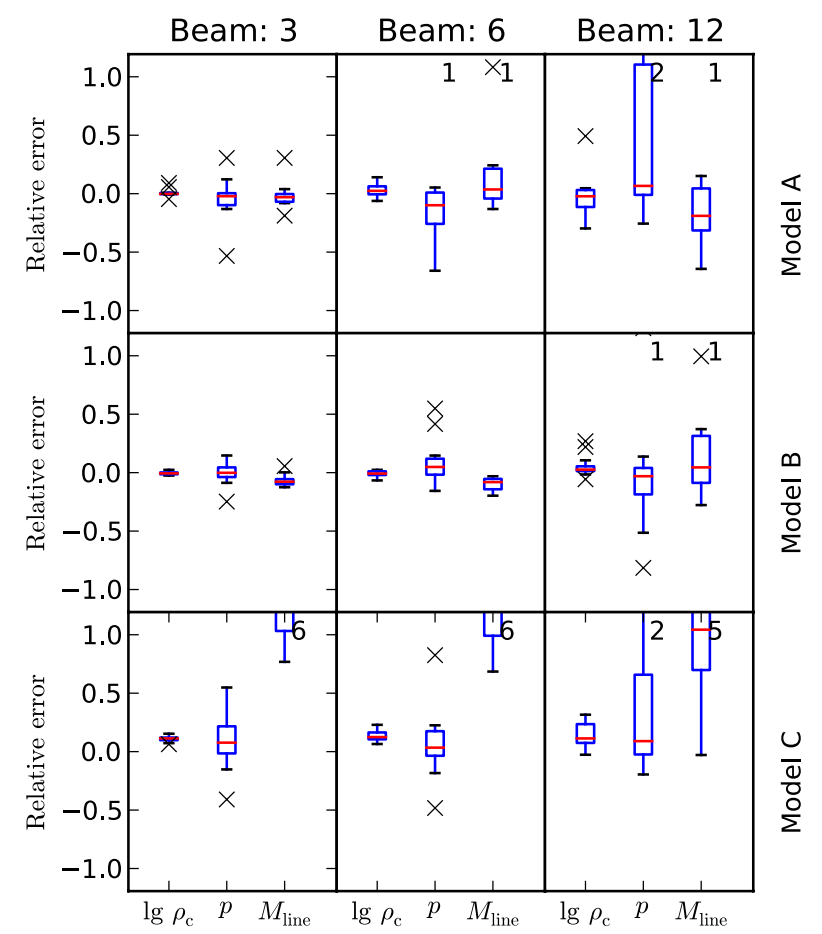

Fig. 11. Boxplot for the relative errors in $\lg \rho_{\mathrm{c}}, p$, and $M_{\text {line }}$ for the three cloud models and the three cloud distances (beam sizes of 3,6 , and 12 pixels corresponding to the near, medium, and far distances). The errors are calculated by comparing the values derived from $N^{\mathrm{Obs}}$ to those obtained from $N^{\text {True }}$ (see Figs. 4, 5, and 8). For each parameter, the box extends between the first and third quartile with a horizontal line indicating the median value. The vertical lines (whiskers) extend from the box to the last data point within a distance equal to 1.5 times the distance between the third and the first quartile. Points outside this range are drawn with crosses. The number of outliers falling outside the plotted range is indicated with numbers near the upper and lower borders.

model clouds and averaged along the length of the projected filament. For model C, the densities have been overestimated because of the difference between the true and the assumed $\kappa_{v}$ values. In models A and B, there is less bias but even in model B the densities are sometimes overestimated by a factor of a few. This is the case for example for the filament number 1 that was already seen to consist of separate density structures along the line of sight. This result reminds us that continuum observations cannot directly measure the volume density. The uncertainty in the $\rho_{\mathrm{c}}$ values is several tens of percent.

Figure 14 also compares the mass estimates $M_{\text {line }}$ with the actual cloud mass within a projected distance of $0.1 \mathrm{pc}$ of the filament centre. Apart from model C, the true masses are on average slightly higher than the values derived from the fits to the filament profiles. To some extent, this may reflect the presence of an extended cloud component that in the Plummer fits is modelled as a separate component and thus not included in the $M_{\text {line }}$ estimates. In some cases, there is a clear anticorrelation between the $\rho_{\mathrm{c}}$ and $M_{\text {line }}$ values (e.g., filaments 4 and 8). When the fitted profile has a narrow peak, a larger fraction of the mass gets attributed to the background component. However, the background is usually low (see Fig. 3) and cannot always explain the factor of $\sim 2$ differences in the models $\mathrm{A}$ and $\mathrm{B}$.

The bias in the mass estimates can be traced back to the assumptions of the column density calculations, i.e., a constant line-of-sight temperature and a dust spectral index $\beta=2.0$

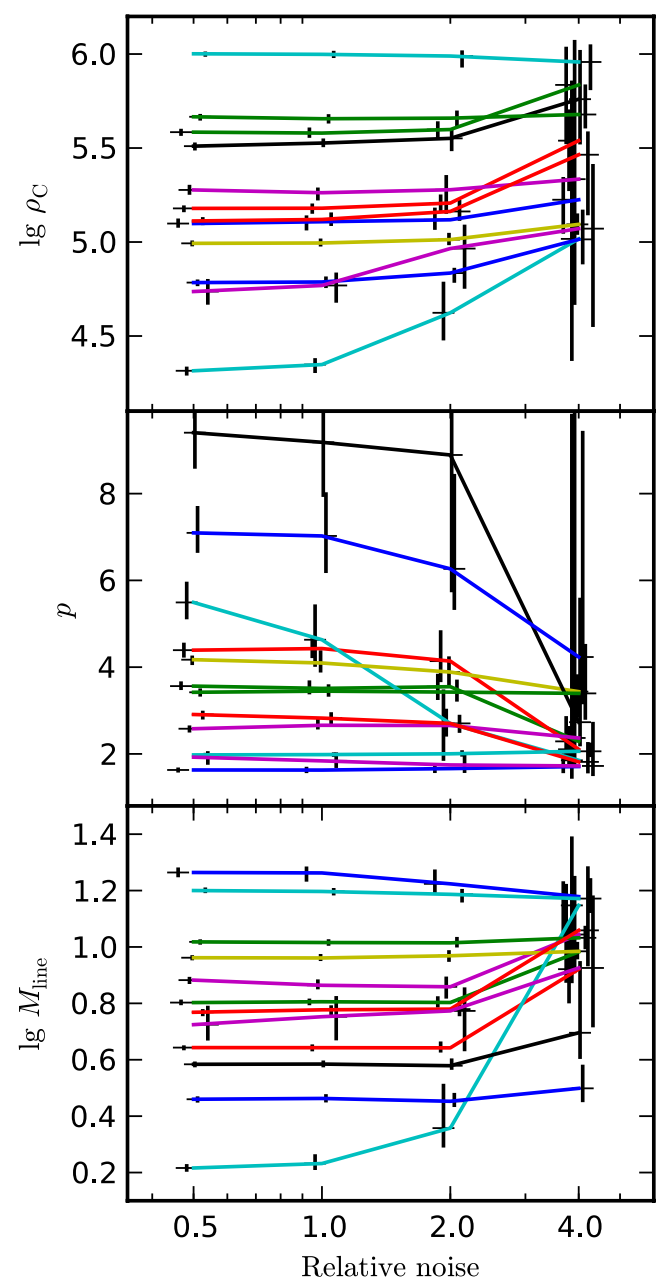

Fig. 12. The dependence of the extracted Plummer parameters $\rho_{\mathrm{c}}, p$, and $M_{\text {line }}$ on the noise level in the case of model A and the near cloud distance. The level of instrumental noise is either 0.5, 1.0, 2.0, or 4.0 times the default value (see Sect. 2). The plot shows for all the twelve filaments the median values and, as vertical bars, the interquartile intervals of the estimated parameters that are obtained from 50 noise realisations of the surface brightness maps. The coloured lines connect the median values. The vertical bars plotted for the individual filaments are shifted slightly along the $x$ axis to avoid overlap.

(instead of the slightly larger actual value of the dust model). Although the bias in the column density values should increase towards the centre of the filament, this was not reflected in the parameters $\rho_{\mathrm{c}}$ or $p$ as a systematic error. In model C, the inclusion of dust with higher sub-millimetre opacity causes a bias mainly in the mass estimates, although the central density and the parameter $p$ should also be affected. That the column densities were overestimated more towards the centre of the filaments should lead to an overestimation of the parameter $\rho_{\mathrm{c}}$. This is also seen in the results, the central densities being overestimated by a factor of 2-3. This is consistent with the modified dust being responsible for most of the signal along the central line of sight. The relative abundance of the dust with higher $\kappa_{v}$ increased as a function of density and this might have been expected to lead to higher $p$ values. In practice, no clear bias, however, is seen. Together with the high column density, the high dust emissivity increased the signal-to-noise ratio in model $\mathrm{C}$ far above that of models A and B. Nevertheless, in model C the scatter in the parameter values was also the largest. 

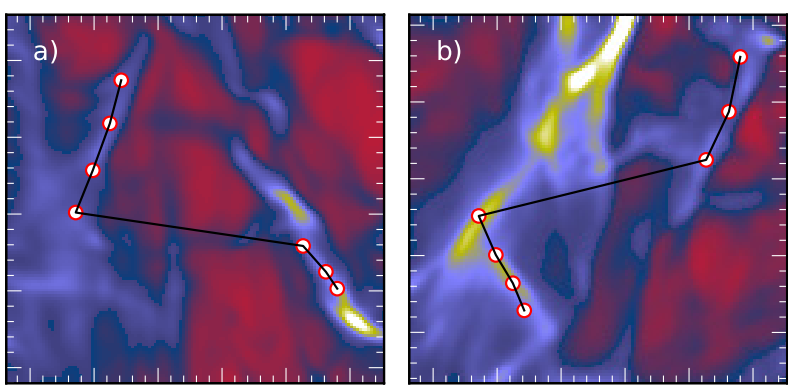

Fig. 13. Filament 1 seen from two orthogonal viewing directions. The object was identified from observations towards the $x$ direction. The figure shows the object from the $y$ and $z$ directions (frames a) and b), respectively). The circles denote volume density maxima for a few lines of sight through the centre of the filament as seen towards the $x$ direction. The background image is the $A_{\mathrm{V}}$ of the model cloud.

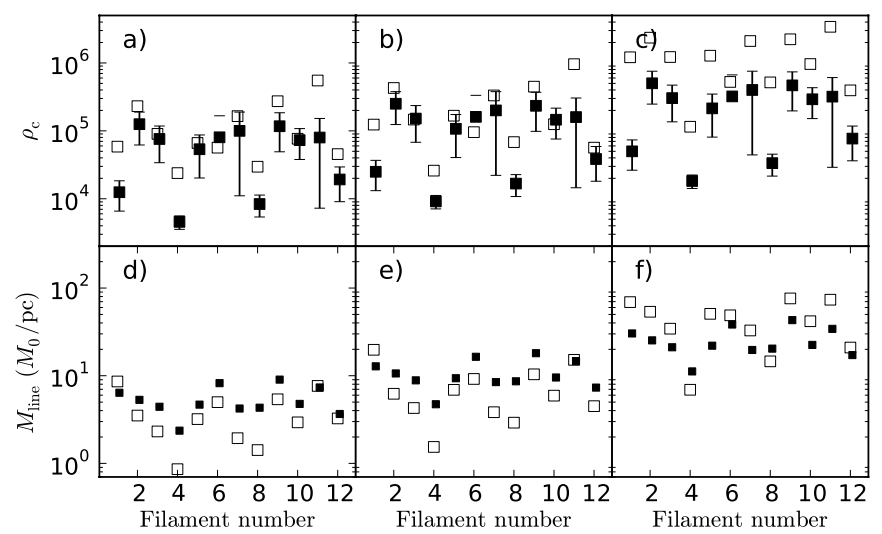

Fig. 14. Comparison of the $\rho_{\mathrm{c}}$ and filament mass estimates from the Plummer fits (open symbols) and the actual values of maximum lineof-sight densities and masses in the cloud model (solid squares). The error bars on the true densities correspond to the $1 \sigma$ dispersion of the peak density values along the filament. In the mass estimates the solid squares correspond to the total mass within a projected distance of $0.1 \mathrm{pc}$ of the filament. Model A: frames a) and d), model B: frames b) and $\mathbf{e}$ ), model C: frames $\mathbf{c}$ ) and $\mathbf{f}$ ).

\section{Conclusions}

We have examined the reliability of the parameters that can be derived for interstellar cloud filaments using dust emission data. The study is based on synthetic observations calculated with both MHD simulations of filamentary clouds and radiative transfer modelling of the sub-millimetre dust emission. The observational parameters correspond to typical Herschel observations. We have examined the estimated FWHM widths of the filaments and the parameters of the Plummer functions used to describe the filament profiles. The results have led to the following conclusions:

- For clouds at a distance of $\sim 100 \mathrm{pc}$, the profile parameters obtained from the observations and from the true column density are usually similar to within a few percent. However, differences of up to tens of percent are observed in the case of a few filaments.
- The results are affected by the instrumental noise. In the higher density models, the signal-to-noise ratios were higher and the errors were correspondingly smaller. If the instrumental noise was increased from the assumed default values, the errors increased rapidly. When the noise was increased by a factor of four, the filament parameters were no longer well-constrained, even for nearby clouds.

- The uncertainties caused by the background confusion and the typical instrumental noise outweigh the effects of radiative transfer.

- The uncertainties increase rapidly when the resolution of the observations is decreased. At a distance of $\sim 400 \mathrm{pc}$, the filaments are barely resolved, the typical errors are larger by a factor of a few, and there are more cases where the profile fit leads to completely wrong parameter values.

- We examined a model where the dust opacity increases as a function of density. In the analysis, the mass estimates were naturally affected by wrong assumptions about the dust opacity. The errors were also larger for the other parameters but they are not strongly biased.

- The derived filament properties are affected by the line-ofsight confusion. In our study, some of the filaments that were identified from the column density maps are discontinuous structures of the 3D density field.

Acknowledgements. The authors acknowledge the support of the Academy of Finland grants Nos. 127015 and 250741. J.M. also acknowledges a grant from Magnus Ehrnrooth Foundation.

\section{References}

André, P., Men'shchikov, A., Bontemps, S., et al. 2010, A\&A, 518, L102 Arzoumanian, D., André, P., Didelon, P., et al. 2011, A\&A, 529, L6 Bally, J., Lanber, W. D., Stark, A. A., \& Wilson, R. W. 1987, ApJ, 312, L45 Draine, B. T. 2003, ApJ, 598, 1017

Elmegreen, D. M., \& Elmegreen, B. G. 1979, AJ, 84, 615

Heitsch, F., Naab, T., \& Walch, S. 2011, MNRAS, 415, 271

Inutsuka, S.-I., \& Miyama, S. M. 1997, ApJ, 480, 681

Juvela, M. 2005, A\&A, 440, 531

Juvela, M., \& Padoan, P. 2003, A\&A, 397, 201

Juvela, M., \& Ysard, N. 2012, A\&A, 539, A71

Juvela, M., Ristorcelli, I., Pagani, L., et al. 2012, A\&A, 541, A12

Köhler, M., Guillet, V., \& Jones, A. 2011, A\&A, 528, A96

Lunttila, T., \& Juvela, M. 2012, A\&A, 544, A52

Malinen, J., Juvela, M., Collins, D. C., Lunttila, T., \& Padoan, P. 2011, A\&A, 530, A101

Malinen, J., Juvela, M., Rawlings, M. G., et al. 2012, A\&A, 544, A50

Mathis, J. S., Mezger, P. G., \& Panagia, N. 1983, A\&A, 128, 212

Men'shchikov, A., André, P., Didelon, P., et al. 2010, A\&A, 518, L103

Meny, C., Gromov, V., Boudet, N., et al. 2007, A\&A, 468, 171

Myers, P. C. 2009, ApJ, 700, 1609

Nutter, D., Kirk, J. M., Stamatellos, D., \& Ward-Thompson, D. 2008, MNRAS, 384,755

Ormel, C. W., Min, M., Tielens, A. G. G. M., Dominik, C., \& Paszun, D. 2011, A\&A, 532, A43

Ossenkopf, V., \& Henning, T. 1994, A\&A, 291, 943

Ostriker, J. 1964, ApJ, 140, 1056

Padoan, P., \& Nordlund, A. 2011, ApJ, 730, 40

Peretto, N., André, P., Könyves, V., et al. 2012, A\&A, 541, A63

Schneider, S., \& Elmegreen, B. G. 1979, ApJS, 41, 87

Stepnik, B., Abergel, A., Bernard, J., et al. 2003, A\&A, 398, 551

Stodólkiewicz, J. S. 1963, Acta Astron., 13, 30

Ysard, N., Juvela, M., Demyk, K., et al. 2012, A\&A, 542, A21 